\title{
Analysis and Treatment for Fatigue Vibration of Instrument Tubes in a PWR Nuclear Power Plant Conventional Island
}

\author{
Shao Jiaye \\ State Nuclear Electric Power Planning Design \& Research Institute Co., Ltd. Beijing, China
}

\begin{abstract}
Several instrument tubes vibrated during the $\mathrm{C}$ stage commissioning period of a PWR nuclear power plant. This essay firstly analyzed the selection of instrument tubes and the velocity of the tubes vibration. Moreover, the author proposed solution towards the tubes vibration which was proved to be effective after applying on the nuclear power plant instrument tubes. The method proposed should be applicable when similar situation reoccurred in other plants.
\end{abstract}

\section{Introduction}

Thermal instruments are important parts of the nuclear power plant automation system and key to ensuring the safety of nuclear power plant operation. In the process of nuclear power plant construction, the installation of instrument tubes lays the foundation for its measurement, controlling system, safe and stable operation.

During the $\mathrm{C}$ stage commissioning period of a domestic pressurized water reactor nuclear power plant, although the vibration did not raise the risk of fatigue fracture, the instrument tubes vibrated several times within the range of the conventional island. Since the instruments are responsible for monitoring the status of the units and some are additionally directly affecting the operating status of the nuclear island and conventional island, the fatigue fraction caused by instrument tubes vibration may lead to unplanned trips or reactor jumps of the unit, and this could lower the safety and economic benefit. Therefore, the instrument tubes should be reinforced to withstand the displacement caused by vibration.

During the start-up and shutdown or load dump test, when the system was under lower power condition, the processing pipe was vibrating intensely due to unstable work medium, and this formed cyclic stress on the instrument tubes connected to the process pipeline. In addition, since the instrument pipe itself was long and flexible, when the stress hit its highest, there should appear cracks at first and finally lead to fatigue fracture if no support was provided to fix the tubes[1].

This paper described the tubes vibration fed back from the nuclear power plant, made evaluation and analysis to instrument tubes itself and its vibration velocity, in additionally, proposed effective vibration reduction measure on the laying path which had already been applied.

\section{Project example}

Event description 1: On November 07, 2018, during the start-up phase of Unit 1 of the nuclear power plant, onsite personnel inspected and found that the main feed water pipe pressure transmitter root valve rear instrument tube vibrated significantly.

Event description 2: On November 13, 2018, the technical department of the nuclear power plant issued a status report. The report described: The air source tube of the condensate recirculation control valve of Unit 2 vibrated obviously, it will affect the condensate recirculation adjustment Valve adjustment of the valve if the tubes break.

\section{Tubes analysis}

The main feed water piping design pressure range is $0 \sim 10 \mathrm{MPa}$. the design temperature range is $0 \sim 228.3^{\circ} \mathrm{C}$. The gauge pipe behind the root valve of the main feed water pipe pressure transmitter is $\varphi 14 \mathrm{~mm} \times 2 \mathrm{~mm}$, and the material is SS316L seamless steel pipe.

The design pressure range of condensate recirculation tube is $0 \sim 4.2 \mathrm{MPa}$. The design temperature range is $0 \sim 138.1^{\circ} \mathrm{C}$. The air source tube of the condensate recirculation regulating valve is $\varphi 12 \mathrm{~mm} \times 2 \mathrm{~mm}$, and the material is SS316L seamless steel pipe.

According to ASME B31.1 [2], regarding the calculation formula for the wall thickness of the instrument tube. Domestic DL/T 5366 [3] stipulated the requirement of $\mathrm{D}_{0}$ (pipe outer diameter)/d (pipe inner diameter) to be not greater than 1.7. Therefore, the instrument tube involved in the above events meets the conditions of use, and the minimum wall thickness of the tube required at the design pressure and design

*Corresponding author's e-mail: shaojyvictor@163.com 
temperature is calculated according to the following formula:

$$
S_{m}=\frac{p D_{0}}{2[\sigma]^{t} \eta+2 Y p}+\alpha
$$

$p$ : Design pressure of medium in tube $(\mathrm{MPa})$;

$D_{0}$ : Outer diameter of tube $(\mathrm{mm})$;

$[\sigma]^{\text {t: }}$ The allowable stress $(\mathrm{MPa})$ of the steel at the design temperature, the corresponding values at $228.3^{\circ} \mathrm{C}$ and $138.1^{\circ} \mathrm{C}$ are $74 \mathrm{MPa}$ and $87 \mathrm{MPa}$ respectively;

$Y$ : Correction factor, the value of stainless steel is 0.4 ;

$\eta$ : Allowable stress correction coefficient, the value of seamless steel pipe is 1.0 ;

$\alpha$. There is an additional thickness of corrosion and abrasion. The corrosion and abrasion of steam and water pipes can be ignored, and the value is 0 .

Considering the influence of tube deviation, the calculated wall thickness of tube pressure is:

$$
S_{C}=S_{m}+c
$$

Sc: Calculated wall thickness of tube $(\mathrm{mm})$;

$c$ : Additional value of negative deviation of tube wall thickness (mm);

Since the above-mentioned instrument tubes are made of seamless steel pipes, the additional value of the negative deviation of the tube wall thickness is calculated according to the following formula:

$$
c=\frac{m}{100-m} S_{m}
$$

$m$ : The allowable negative deviation (\%) of the wall thickness specified in the technical conditions of tube products is generally $15 \%$

In the event description 1 , let the design pressure to be its maximum $10 \mathrm{MPa}$, the temperature to be its maximum $228.3^{\circ} \mathrm{C}$, and the calculated wall thickness to be $1.05 \mathrm{~mm}$ to meet the pressure requirements of the tube. SS316L, $\varphi 14 \mathrm{~mm} \times 2 \mathrm{~mm}$ tubes were selected to meet the above requirements. In the event description 2 , let the design pressure to be its maximum $4.2 \mathrm{MPa}$, the temperature to be its maximum $138.1^{\circ} \mathrm{C}$, and the calculated wall thickness to be $0.24 \mathrm{~mm}$ to meet the pressure requirements of the tube. SS316L, $\varphi 12 \mathrm{~mm} \times 2 \mathrm{~mm}$ tubes were selected to meet the requirements.

\section{Vibration velocity evaluation analysis}

The start-up and shutdown of the unit and the vibration of the process pipeline due to the pulsating impact of the fluid medium would cause the instrument tube connected to vibrate. The vibration velocity of the instrument tube was measured and evaluated according to the domestic adopted DL/T 1103 [4] standard. By continuously measuring the vibration velocity at different points of the tube to determine the location of the maximum vibration velocity, the maximum peak velocity $\left(\boldsymbol{v}_{\text {max }}^{\text {peak }}\right)$ was detected and compared with the maximum allowable peak velocity $\left(\boldsymbol{v}_{\text {allow }}^{\text {peak }}\right.$ ). The allowable peak velocity is calculated according to the following formula:

$$
v_{\text {allow }}^{\text {peak }}=\frac{13.4 C_{1} C_{4}}{C_{3} C_{5}} \frac{S_{e l}}{\alpha C_{2} K_{2}}
$$

13.4: Conversion factor, $\mathrm{mm} / \mathrm{s} / \mathrm{MPa}$;

$C_{l}$ : Compensate the correction coefficient of the concentrated mass on the characteristic tube, take the value according to Fig.1;

$C_{4}$ : End condition correction factor;

$S_{e l}: 0.8 \mathrm{~S}_{\mathrm{A}}$, where $\mathrm{S}_{\mathrm{A}}$ is based on the alternating stress specified in Section III of the ASME BPV code[5]. In this paper, the maximum allowable alternating stress of $10^{11}$ cycles is selected, and the value of stainless steel pipe is $114 \mathrm{MPa}$;

$C_{3}$ : The correction coefficient of the medium and insulation layer in the tube, because this part of the instrument tube has no insulation layer, the value here is 1 ;

$W$ : The mass of the pipe per unit length, $\mathrm{kg} / \mathrm{m}$;

$W_{F}$ : The mass of the medium in the unit length of the tube, $\mathrm{kg} / \mathrm{m}$;

$W_{I N S}$ : The mass of the insulation layer per unit length of the tube, $\mathrm{kg} / \mathrm{m}$;

$C_{5}$ : Consider the correction factor for forced vibration to deviate from resonance;

$\mathrm{C}_{2}$ : Secondary stress index, the value specified in Section III of the ASME BPV code;

$K_{2}$ : Local stress index, the value specified in Section III of ASME BPV code;

$\alpha$. The allowable stress reduction factor is 1.3 for the material included in Section III Figure I-9.1 of the ASME BPV Code; or 1 for the material included in FigI9.2.1 or Fig I-9.2.2 of the ASME BPV Code Section III. When each parameter takes conservative values, for stainless steel tube, $\left(v_{\text {allow }}^{\text {peak }}\right)=21.3 \mathrm{~mm} / \mathrm{s}$.

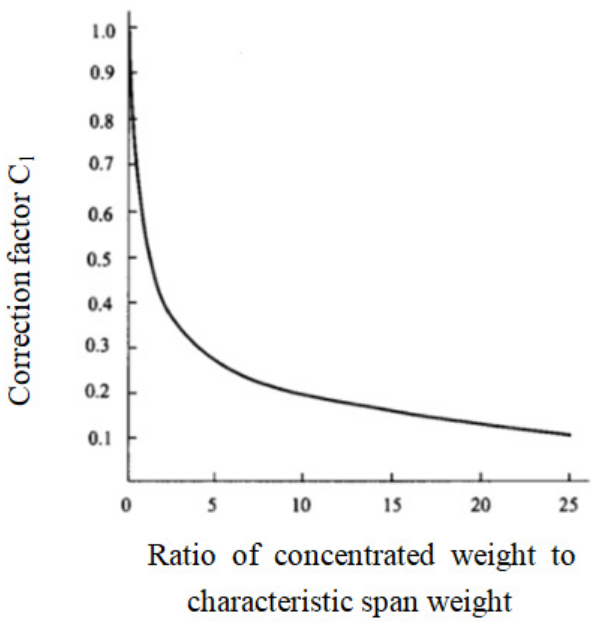

Fig.1 The value of correction factor $\mathrm{C} 1$

The evaluation method of vibration speed of stainless steel pipe is shown in Tab.1.

Tab.1 The velocity method of vibration of stainless steel pipe

\begin{tabular}{ccc}
\hline $\begin{array}{c}\text { Maximum peak vibration } \\
\text { velocity } \\
\text { measurement } v_{\text {max }}^{\text {peak }}(\mathrm{mm} / \mathrm{s})\end{array}$ & $\begin{array}{c}\text { Vibration } \\
\text { quality }\end{array}$ & $\begin{array}{c}\text { Assessment } \\
\text { requirements }\end{array}$ \\
\hline $0 \leq v_{\text {max }}^{\text {peak }} \leq 21.3$ & Excellent & N/A
\end{tabular}




\begin{tabular}{ccc}
$21.3 \leq v_{\text {max }}^{\text {peak }} \leq v_{\text {allow }}^{\text {peak }}$ & Qualified & Track \\
$v_{\text {max }}^{\text {peak }} \geq v_{\text {allow }}^{\text {peak }}$ & Assessment & Evaluation \\
$v_{\text {max }}^{\text {peak }}>2 v_{\text {allow }}^{\text {peak }}$ & Unacceptable & Process \\
\hline
\end{tabular}

According to the vibration data of the nuclear power plant $1 \#$ unit measured in the startup process on November 7 , the unit was at a $25 \%$ thermal power platform and the maximum velocity of the main water supply pipe where the measuring instrument tube located was $31.07 \mathrm{~mm} / \mathrm{s}$ which was lower than the allowable peak velocity of $36.03 \mathrm{~mm} / \mathrm{s}$. Thus, the vibration of the pipe system meets the requirements.

Under the condition of the $2 \#$ unit of the nuclear power plant at $65 \%$ thermal power platform on November 13, the maximum velocity of the condensate recirculation pipe where the gas source pipe measured was $23.83 \mathrm{~mm} / \mathrm{s}$ which was less than the allowable peak velocity of $27.76 \mathrm{~mm} / \mathrm{s}$. Thus, the vibration of the pipe system meets the requirements.

\section{Plan and result}

\subsection{Design}

Since the current vibration observed only existed under the operating condition of the start-up phase and the vibration qualities of the two engineering examples were both qualified according to the above analysis, the local support of the instrument tube can be improved, the vibration of the instrument tube and the displacement can be reduced by adding brackets. The two design schemes given are shown in Fig.2 (a) and (b). The setting principles of the design was to find the fixed support point, set the sliding support point, then to add the support accordingly: when the seamless steel pipe was laid horizontally the distance between the supports should be $1200 \mathrm{~m}$ where when laid vertically the distance between the supports should be $1500 \mathrm{~mm}$.

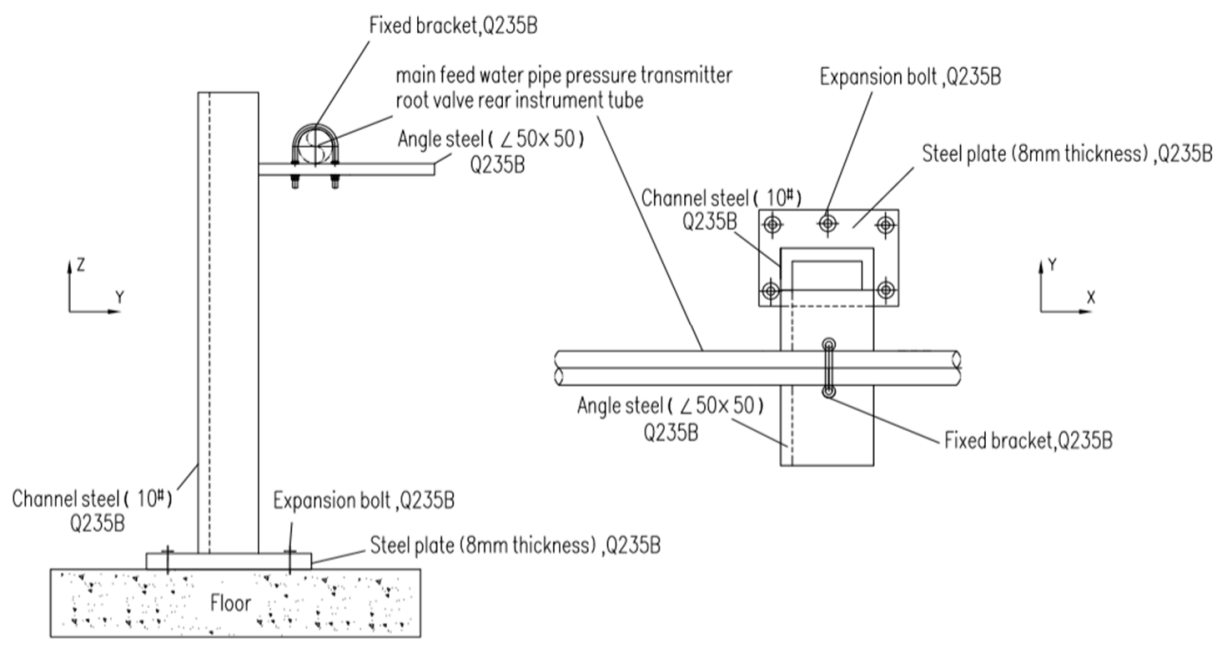

(a)

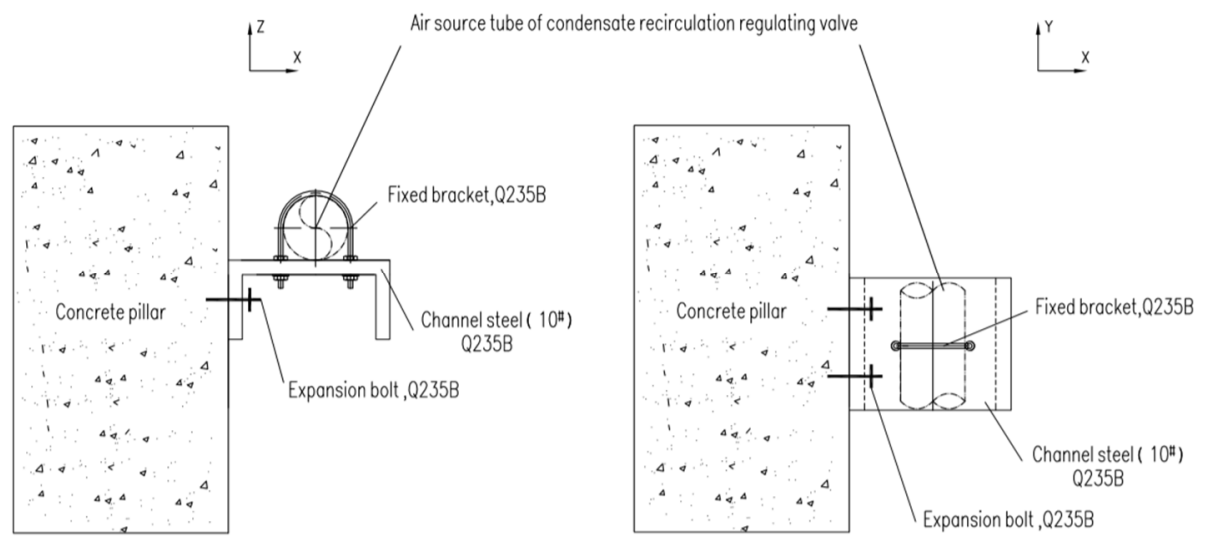

(b)

Fig.2 Vibrating tubes support design 


\subsection{Implementation result}

After the plan was confirmed by the on-site units, it was implemented through the minor repair window of the unit by implementing on-site with retest. The vibration reduction was obvious with the maximum velocity between $0-21.3 \mathrm{~mm} / \mathrm{s}$. Figure 3 (a) and (b) are photos of on-site implementation results.

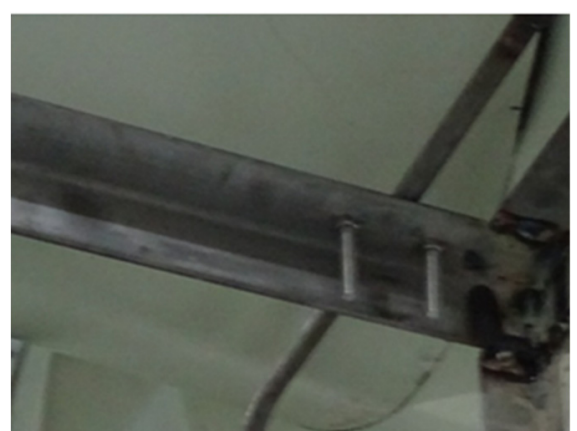

(a)

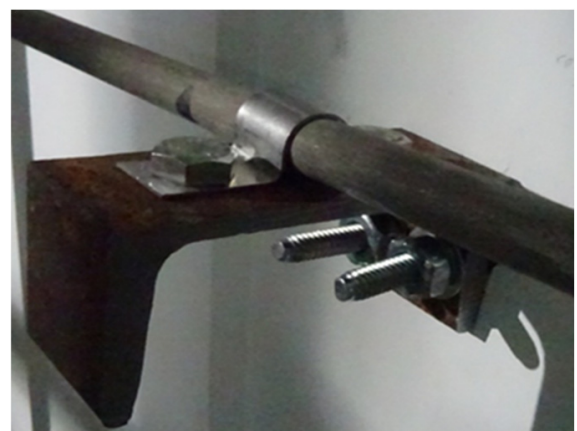

(b)

Fig.3 Implementation result photos

\section{Conclusions and suggestions}

The following conclusions can be obtained through the analysis and evaluation of the vibrating tube and the analysis of peak velocity:(1) The thickness of the tube wall and the material of the tube met the pressure requirements; (2) The maximum velocity was less than the allowable peak velocity, although visible amplitude was observed, no pipeline fracture incurred. The pipeline was fixed by adding reasonable support, and with on-site actual inspection, the results were proved feasible.

Suggestions: (1) When the laying length of the straight pipe section is greater than 3 times the maximum distance, a fixed bracket should be set. For seamless steel pipes laid horizontally, the distance between the brackets could be $1000 \mathrm{~mm}$ to $1500 \mathrm{~mm}$. As for vertical laying, the distance between the brackets could be $1500 \mathrm{~mm}$ to 2000mm; (2) The bracket should be detachable, matching the pipe diameter and be firmly fixed. The fixed end of the bracket should not be set on the pressure vessel or detachable equipment.(3) By increasing the diameter and wall thickness of the instrument tube appropriately to increase the natural frequency of the structure, the influence of low-frequency vibration can be improved.(4) For the connection of pipe fittings of different sizes, reducer joints can be used to reduce local stress and improve connection reliability. (5) According to the feedback on the site, although the vibration did not cause the risk of fracture, the follow-up requires on-site maintenance personnel to pay more attention to the parts of the instrument pipeline connection. Effective welding can change the strength of the structural connection and strengthen the investigation of the weld at the same time.

\section{References}

1. Prokofiev A, Makariyants G, Shakhmatov E. Modeling of pipeline vibration under the pressure ripples in the working fluid $[\mathrm{C}] / /$ Proceedings of the 17 th International Congress on Sound and Vibration. 2010 .
2. ASME B31.1-2016 Power Piping [S].2016.

3. DL/T 5366-2014 Technical Code for Stress Calculating of Steam/Water Piping in Fossil Fuel Power Plant [S]. China Electric Power Press, 2014.

4. DL/T 1103-2009 Nuclear Power System Pipe System Vibration Test and Evaluation [S]. China Electric Power Press,2009.

5. ASME BPV Section III, Boiler and Pressure Vessel Criteria. Vol.3[S]. 